\title{
DALI: Defining Antibiotic Levels in Intensive care unit patients: a multi-centre point of prevalence study to determine whether contemporary antibiotic dosing for critically ill patients is therapeutic
}

\author{
Jason A Roberts ${ }^{1,2^{*}}$, Jan J De Waele ${ }^{3}$, George Dimopoulos ${ }^{4}$, Despoina Koulenti ${ }^{4}$ Claude Martin $^{5}$, \\ Philippe Montravers ${ }^{6}$, Jordi Rello ${ }^{7}$, Andrew Rhodes ${ }^{8}$, Therese Starr ${ }^{2}$, Steven C Wallis ${ }^{1}$ and Jeffrey Lipman ${ }^{1,2}$
}

\begin{abstract}
Background: The clinical effects of varying pharmacokinetic exposures of antibiotics (antibacterials and antifungals) on outcome in infected critically ill patients are poorly described. A large-scale multi-centre study (DALI Study) is currently underway describing the clinical outcomes of patients achieving pre-defined antibiotic exposures. This report describes the protocol.

Methods: DALI will recruit over 500 patients administered a wide range of either beta-lactam or glycopeptide antibiotics or triazole or echinocandin antifungals in a pharmacokinetic point-prevalence study. It is anticipated that over 60 European intensive care units (ICUs) will participate. The primary aim will be to determine whether contemporary antibiotic dosing for critically ill patients achieves plasma concentrations associated with maximal activity. Secondary aims will compare antibiotic pharmacokinetic exposures with patient outcome and will describe the population pharmacokinetics of the antibiotics included. Various subgroup analyses will be conducted to determine patient groups that may be at risk of very low or very high concentrations of antibiotics.
\end{abstract}

Discussion: The DALI study should inform clinicians of the potential clinical advantages of achieving certain antibiotic pharmacokinetic exposures in infected critically ill patients.

Keywords: Antibiotic, ßeta-lactam, Glycopeptide, Triazole, Echinocandin, Continuous infusion, Extended infusion, Bolus dosing, Adverse events, Pharmacokinetics, Pharmacodynamics

\section{Background}

Effective antibiotic treatment of intensive care unit (ICU) patients that have overwhelming infections, including sepsis, severe sepsis and septic shock, remains a significant challenge to physicians world-wide [1-7]. Therapy that is not initiated rapidly or with sufficient antibiotic spectrum increases in-hospital mortality [3,4]. Indeed, sepsis itself has an incidence in the population that exceeds colon

\footnotetext{
* Correspondence: j.roberts2@uq.edu.au

'Burns Trauma and Critical Care Research Centre, The University of Queensland, Brisbane, Australia

${ }^{2}$ Royal Brisbane and Women's Hospital, Brisbane, Australia

Full list of author information is available at the end of the article
}

cancer, breast cancer, and AIDS, with mortality rates of $30 \%$ for mild to moderate sepsis and up to $82 \%$ for severe sepsis and septic shock [5]. Despite advances in critical care medicine, the incidence of sepsis continues to increase and the prognosis remains poor. Although there has been significant investment into treatments that limit the various inflammatory and coagulation cascades, none of these therapies have been able to demonstrate the same outcome benefits as effective antibiotic therapy [3,8]. It is thought that optimisation of antibiotic dosing may well further improve clinical outcomes for ICU patients with infections. 
Drug doses are usually derived from healthy volunteers and then extrapolated into ICU patients. A challenge for clinicians is that standard dosing in ICU patients does not achieve the same concentrations seen in noncritically ill patients [9]. There is a significant body of literature that demonstrates that disease-processes experienced by critically ill patients frequently cause pharmacokinetic changes that may result in either sub-therapeutic or toxic drug concentrations of antibiotics (including antibacterial and antifungal drugs) [9-17]. Given the increased level of resistance of bacteria in the ICU [18], and these potentially lower antibiotic exposures, treatment failure of infections is unsurprisingly common. Subtherapeutic drug concentrations may also promote selection of resistant microorganisms, further adding to the threat of antibiotic resistance in the ICU. To address this issue innovative approaches to dosing may be required to ensure optimal drug exposures [19-21].

Although altered antibiotic concentrations have been accurately described in various critically ill patient subpopulations in small research studies [21-28], there is no large multi-centre evaluation that seeks to determine whether the issues identified in a controlled research environment correspond to clinical practice. Such an evaluation is essential for determining whether action is required to change existing global antibiotic prescribing practices for critically ill patients. If prescribing should be found to be sub-optimal, then the motivation for changes to existing prescribing practice may lead to improved clinical cure rates and a reduction in the rate of antibiotic resistance in the critical care environment. To address the insufficiency of data available to clinicians on the adequacy of empiric antibiotic dosing in ICU patients, on behalf of the Infection Section of the European Society of Intensive Care Medicine, the authors proposed a multicentre point-prevalence pharmacokinetic study in ICU patients.

This proposal has been supported by the European Society of Intensive Care Medicine's European Critical Care Research Network (ESICM ECCRN) and Trials Group and also the the Royal Brisbane and Women's Hospital Research Foundation (Australia). This report describes the study protocol.

Based on our previous research in many different critically ill patient sub-populations, we hypothesize that 40 to $70 \%$ of critically ill patients are receiving suboptimal antibiotic dosing [23,27-33].

\section{Methods/Design}

The Defining Antibiotic Levels in Intensive care unit patients (DALI) study is a prospective, multi-centre pharmacokinetic point-prevalence study describing whether contemporary antibiotic dosing in ICU patients achieves concentrations associated with maximal activity. It is anticipated that the study will recruit over 500 ICU patients from over 60 ICUs throughout 10 countries in Europe over a one-week period. The primary and secondary aims as well as the proposed sub-group analyses are as follows:

Primary aim

- To determine whether contemporary antibiotic dosing for critically ill patients achieves concentrations associated with maximal activity.

Secondary aims

- Comparison of observed antibiotic pharmacokinetics/pharmacodynamics with the clinical outcome of therapy

- Description of the population pharmacokinetics of the individual antibiotics in ICU patients

The proposed subgroups for the primary and secondary aims are:

- Patients administered intermittent dosing versus extended or continuous infusions and

- Patients with 'steady-state' versus 'non-steady-state' pharmacokinetics ('non-steady-state' defined as antibiotics commenced within 24-h prior to sampling)

- Patients with different levels of sickness severity as measured by Sequential Organ Failure Assessment (SOFA) Score[34], Acute Physiology and Chronic Health Evaluation (APACHE) II Score[35] and PIRO (Predisposition, Infection, Response, Organ dysfunction) Score[36]

- Different admission diagnoses

- Different indications for antibiotic therapy

- Presence of surgery within the 24-hours prior to sampling

- Different total body weight

- Different levels of renal function and presence of extracorporeal renal support techniques

\section{Participants}

Identification of eligible patients will occur on a designated day (preferably Monday) of a nominated week. Informed consent is required from each patient or a legally authorised representative to participate in the study. Participants would need to fulfil all the inclusion and exclusion criteria to be enrolled:

Inclusion criteria

- Written informed consent has been obtained from the patient or their legally authorised representative 
- Age $\geq 18$ years

- Receiving antibiotic therapy of one of the target drugs via continuous or intermittent dosing regimen

- Suitable intravenous/intra-arterial access to facilitate sample collection

\section{Exclusion criteria}

- Consent not obtained

- Aged $<18$ years of age

- Not being administered any of the study antibiotics

- Limited or no intravenous/intra-arterial access.

\section{Study treatments and pharmacokinetic sampling}

With the exception of blood sampling, there is no intervention in this study that may affect patient treatment. Antibiotic dosing will occur as deemed by the treating clinician and their local dosing practices. Patients receiving the study antibiotics will be identified on the nominated week for pharmacokinetic sampling. During a single dosing interval of that week, each patient will then have two blood samples taken for each antibacterial agent and/or three blood samples for antifungals (Table 1). For patients on multiple study drugs, each drug will be sampled independent of the other drug(s). Table 1 outlines the test antibiotics (antibacterials and antifungals) to be sampled, the timing of pharmacokinetic sampling and the pharmacodynamics endpoints that will be tested for each antibiotic and dosing regimen.

The blood sampling has been designed to determine drug concentrations at various time points to describe whether pharmacokinetic/pharmacodynamic targets are achieved in individual patients (Table 1). To achieve the endpoint $50 \% f \mathrm{~T}_{>\mathrm{MIC}}$, sample $\mathrm{A}$ is taken mid-way through the dosing interval to see if the drug concentration exceeds the MIC. The $100 \% f \mathrm{~T}_{>\mathrm{MIC}}$ endpoint is similarly assessed from the sample B taken at the end of the dosing interval. The endpoint $100 \% f \mathrm{~T}_{>4 \times M I C}$, is attained if all sample concentrations exceed the MIC by at least a factor of four. For the parameter $\mathrm{AUC}_{0-24} /$ MIC, the Area Under the concentration-time Curve from $0-24 \mathrm{~h}\left(\mathrm{AUC}_{0-24}\right)$ is calculated by the trapezoidal rule, and to attain the stated endpoint, the $\mathrm{AUC}_{0-24}$ must exceed the MIC by the factor listed in Table 1. Where the MIC not known, the MIC of the infecting pathogen will be defined by The European Committee on Antimicrobial Susceptibility Testing (EUCAST) $\mathrm{MIC}_{90}$ data; available at: http://www. eucast.org/clinical_breakpoints).

\section{Data collection and management}

Data collection will be conducted by trained staff at each participating centre and entered onto a case report form

Table 1 Study drugs, routes of administration, pharmacokinetic/pharmacodynamic targets and blood sampling

\begin{tabular}{|c|c|c|}
\hline Study Drugs and method of infusion & Pharmacokinetic Sampling & $\begin{array}{l}\text { Pharmacodynamic } \\
\text { Targets tested }\end{array}$ \\
\hline Beta-lactam antibiotics by intermittent infusion & $\begin{array}{l}\text { Sample A: mid-way through dosing interval } \\
\text { (50\% of dosing interval) }\end{array}$ & $\cdot 50 \% f \mathrm{~T}_{>\mathrm{MIC}}$ \\
\hline $\begin{array}{l}\text { (amoxycillin-clavulanate; ampicillin; piperacillin-tazobactam; } \\
\text { penicillin-G; flucloxacillin; dicloxacillin; cloxacillin; cephazolin; } \\
\text { ceftazidime; ceftriaxone; cefepime; meropenem; imipenem; } \\
\text { doripenem; ertapenem) }\end{array}$ & $\begin{array}{l}\text { Sample B: within } 30 \text { min of next dose } \\
\text { (100\% of dosing interval) }\end{array}$ & $\cdot 50 \% f \mathrm{~T}_{>4 \times M I C} C^{*}$ \\
\hline \multirow{4}{*}{$\begin{array}{l}\text { Glycopeptide antibiotics by intermittent infusion } \\
\text { (vancomycin, teicoplanin) }\end{array}$} & & $\cdot 100 \% f T_{>M I C}$ \\
\hline & & $\cdot 100 \% f T_{>4 \times M I C}$ \\
\hline & & $\cdot 100 \% f T_{>4 \times M I C}$ \\
\hline & & - Concentration $\geq 15 \mathrm{mg} / \mathrm{L}^{*}$ \\
\hline Triazole antifungals (fluconazole, voriconazole) & $\begin{array}{l}\text { Sample A: } 30 \text { min after completion of intravenous } \\
\text { infusion (peak concentration) }\end{array}$ & $\cdot \mathrm{AUC}_{0-24} / \mathrm{MIC} \geq 25^{*}$ \\
\hline \multirow[t]{2}{*}{ Echinocandin antifungals (caspofungin, micafungin, anidulafungin) } & $\begin{array}{l}\text { Sample B: mid-way through dosing interval } \\
\text { (50\% of dosing interval) }\end{array}$ & $\cdot \mathrm{AUC}_{0-24} / \mathrm{MIC} \geq 20^{*}$ \\
\hline & $\begin{array}{l}\text { Sample C: within } 30 \text { min of next dose } \\
\text { (100\% of dosing interval) }\end{array}$ & \\
\hline Beta-lactam antibiotics (listed above) by continuous infusion & Sample A: at any time & $\cdot 100 \% f \mathrm{~T}_{>4 \times M I C}{ }^{*}$ \\
\hline \multirow[t]{2}{*}{ Glycopeptide antibiotics (listed above) by continuous infusion } & Sample B: $>6$ hours after sample A & $\cdot 100 \% f \mathrm{~T}_{>4 \times \mathrm{MIC}}{ }^{*}$ \\
\hline & & $\cdot \mathrm{AUC}_{0-24} / \mathrm{MIC} \geq 350$ \\
\hline
\end{tabular}

\footnotetext{
* denotes the primary endpoint - other stated pharmacodynamics targets are secondary endpoints.
}

$f \mathrm{~T}_{>\mathrm{MIC}}$ is the duration of a dosing interval for which the antibiotic concentration remains above the minimum inhibitory concentration (MIC) of the known or suspected pathogen (endpoints of $50 \%$ or $100 \%$ of the interval, and MIC is defined by EUCAST MIC ${ }_{90}$ data); $f \mathrm{~T}_{>4 \times \mathrm{MIC}}$ is the duration of a dosing interval for which the antibiotic concentration remains above a concentration that is $4 \mathrm{x}$ the MIC of the known or suspected pathogen (endpoints of $50 \%$ or $100 \%$ of the interval, and MIC is defined by EUCAST MIC 90 data); $\mathrm{AUC}_{0-24} / \mathrm{MIC}$ is the ratio of the area under the concentration time curve from $0-24$ hours to MIC. 
(CRF). At the end of the patient's participation, the CRF will be sent to the coordinating centre (The University of Queensland, Australia). Outstanding queries regarding the completion of the CRF will be undertaken with each participating centre where necessary to ensure accuracy of data.

The data to be collected includes.

Demographic data

- Age

- Gender

- Height

- Weight

Clinical data

- Admission diagnosis,

- Sickness severity scores (APACHE II, SOFA, PIRO)

- Presence of extracorporeal circuits (e.g. RRT (renal replacement therapy), ECMO (extracorporeal membrane oxygenation))

- Procalcitonin (where available),

- Presence/absence of surgery within previous 24 hours

- Clinical outcome of infection

- Mortality at 30-days

Organ function data

- Renal function - serum creatinine concentration during studied dosing interval; MDRD (modified diet in renal disease) equation

- 8-hours urinary creatinine clearance (where available)

- Fluid balance for total length of stay and previous 24-hours

Antibiotic dosing data

- Dose and frequency

- Time of dosing and sampling

- Day of antibiotic therapy

Infection data

- Known or presumed pathogen

- Known or likely minimum inhibitory concentration (MIC)

The definitions used to assess clinical outcome of therapy are as follows. A positive clinical outcome of therapy is defined as completion of treatment course without change or addition of antibiotic therapy, and with no additional antibiotics commenced with $48 \mathrm{~h}$ of discontinuation of the antibiotic therapy.

De-escalation is defined as the change to a narrower spectrum antibiotic based on patient-specific microbiological data in the absence of clinical failure. For antibiotics that are ceased before the end of treatment of an infection because of antibiotic de-escalation, these antibiotics will be excluded from the $a$ priori analysis. Where deescalation occurs during the target week of sampling, an option for a second sampling period of the new antibiotic will exist to confirm appropriateness of antibiotic concentrations and compare these with the patient's clinical outcome.

Safety data will be collected to define any adverse drug reaction (clinically observed, haematological or biochemical) that is reported by the clinical staff at the participating ICUs that is suspected as being caused by any of the study antibiotics.

\section{Maintenance of blood sample integrity}

Blood samples will be kept on ice, centrifuged at $3000 \mathrm{rpm}$ for 10-min, within $6 \mathrm{~h}$ of collection and the plasma transferred to a labelled cryo-vial for frozen storage (at $-20^{\circ} \mathrm{C}$ or lower for short term storage). A commercial courier company specialising in transport of clinical samples on dry ice will collect the samples from each site and deliver to the Burns Trauma and Critical Care Research Centre at The University of Queensland, Australia for bioanalysis. Samples will be stored at $-80^{\circ} \mathrm{C}$ until assay.

\section{Bioanalysis}

The concentration of the study antibiotics in the biological samples will be determined by chromatographic methods (HPLC and LC-MS/MS) that are validated and conducted in accordance with the US Food and Drug Administration's guidance for industry on bioanalysis (available at: www.fda.gov/downloads/Drugs/GuidanceCompliance RegulatoryInformation/Guidances/UCM070107.pdf).

\section{Ethical issues}

Each of the participating centres has obtained local ethics approvals to conduct the study as described in Additional file 1. The University of Queensland is the head institution with ethical approval granted by the Medical Research Ethics Committee (201100283 12th April 2011 and Amendment 201100283 25th May 2011). Patients may withdraw from the study at any time without prejudice, as documented and explained at the time of consenting.

\section{Statistical and pharmacokinetic analysis}

The achievement of the pharmacodynamics targets will be performed by visual inspection of the results and comparison with the target. Statistical analyses to test the 
study objectives will be performed using Mann-Whitney $\mathrm{U}$ tests or Students t-tests where appropriate using the statistical package, SPSS (version 17.0, Illinois, USA).

The $\% f \mathrm{~T}_{>\mathrm{MIC}}$ will be determined using the equation [37]:

$$
\% f T_{>\text {MIC }}=\ln \left[\text { Dose } /\left(V_{\mathrm{d}} \times \mathrm{MIC}\right)\right] \times\left(1 / k_{e l}\right) \times(100 / \mathrm{DI})
$$

where $V_{d}$ is volume of distribution calculated as Dose/ AUC; MIC is the known or suspected minimum inhibitory concentration; kel is the elimination rate constant calculated from the gradient of the concentration-time curve in the elimination phase (sample A and sample B) and DI is the dosing interval (h). Where infections are polymicrobial, the MIC of the least susceptible pathogen will be used in the analysis.

The population pharmacokinetic parameters of each antibiotic will be determined using a population pharmacokinetic modelling approach using NONMEM $^{\bullet}$ (Version 6.1, GloboMax LLC, Hanover, MD, USA) as previously described[22,23,25,29,32,38]. Additionally, the pharmacokinetic model will aim to determine if significant correlations exist between demographic and clinical factors on pharmacokinetics. If one or more of the variables are found to have a significant effect on the pharmacokinetics of the drug, then it can be incorporated into the final pharmacokinetic model.

\section{Sample size and power}

Whilst it is not possible to predict the number of patients receiving each of the study antibiotics, all data will be useful and can be used to inform clinical practice. It is likely that at least 5 of the study antibiotics will have a minimum of 30 patients included in the analysis. This sample size will provide a power of $80 \%$ (assuming an $\alpha$ of 0.05 and $r^{2}$ of $30 \%$ ) for defining at least 2-4 covariates predictive of achieving the primary pharmacodynamic outcome [39]. For all other included study antibiotics, we estimate that each will have a minimum of 12 patients that can be used for the secondary objectives of population pharmacokinetic analysis. A minimum of 12 patients per antibiotic is based on data from previous non-interventional pharmacokinetic studies in critically ill patients [22,23,32,40].

\section{Funding}

This project has received funding from the European Society of Intensive Care Medicine's European Critical Care Research Network (ESICM ECCRN), and the Royal Brisbane and Women's Hospital Foundation. Dr Roberts is funded by a Training Research Fellowship from the National Health and Medical Research Council of Australia (569917).

\section{Discussion}

ICU patients are greatly different in many ways to noncritically ill patients. Principal differences relate to the level of sickness severity, the number of therapeutic interventions used, the severe pathophysiological changes that occur and the presence of highly resistant bacteria and fungi. For these reasons, it unsurprising that ICU patients have poor outcomes associated with infections. Whilst early and appropriate treatment of infections significantly reduces patient mortality, the additional benefits of optimised antibiotic pharmacokinetic exposures have been poorly quantified. ICU pharmacokinetic studies have traditionally only enrolled small patient numbers, which greatly limits the ability to describe the significant interpatient pharmacokinetic variability that is present, and what effect this may have on clinical efficacy. Using a multinational approach to enrol large patients numbers on a wide range of commonly used antibiotics, including both antibacterials and antifungals, the DALI study will address these knowledge gaps.

\section{Additional file}

Additional file 1: The location of the participating sites (country and city) and Ethics committee approving conduct of the study in each site.

\section{Abbreviations}

AIDS: Acquired Immunodeficiency syndrome; APACHE: Acute physiology and chronic health evaluation; AUC: Area under the concentration-time curve; $\mathrm{AUC}_{0-22} / \mathrm{MIC}$ : Area under the concentration-time curve from 0-24 h; CRF: Case report form; DALI: Defining Antibiotic Levels in Intensive care patients; DI: Dosing interval; ECCRN: European Critical Care Research Network; ECMO: Extracorporeal membrane oxygenation; ESICM: European Society of Intensive Care Medicine'; $f T_{>\text {MIC: }}$ The duration of a dosing interval for which the antibiotic concentration remains above the MIC of the known or suspected pathogen; $f T_{>4 \times M M l}$ : The duration of a dosing interval for which the antibiotic concentration remains above a concentration that is $4 \times$ the MIC of the known or suspected pathogen; HPLC: High performance liquid chromatography; ICU: Intensive care unit; kel: Elimination rate constant; LCMS/MS: Liquid chromatography tandem mass spectrometry; MDRD: Modified diet in renal disease; MIC: Minimum inhibitory concentration;

$\mathrm{MIC}_{90}$ : Concentration required to inhibit the growth of $90 \%$ of organisms after 24-h; PIRO: Predisposition, infection, response, organ dysfunction; RRT: Renal replacement therapy; SOFA: Sequential organ failure assessment; $V_{d}$ : Volume of distribution.

\section{Competing interests}

The authors declare that they have no competing interests.

\section{Authors' contribution}

$J A R$ and $J L$ designed the study and wrote the initial protocol. JJD, GD, DK, CM, PM, JR, AR TS and SCW TS provided advice and input into the protocol. All authors read and approved the final manuscript.

This study was partly funded by the European Society of Intensive Care Medicine from which it received a grant (see below).

\section{Acknowledgements}

The authors wish to acknowledge the administrative support provided by Ms Renae Deans (The University of Queensland, Brisbane, Australia) for her help in preparing the protocol. 


\section{Author details}

${ }^{1}$ Burns Trauma and Critical Care Research Centre, The University of Queensland, Brisbane, Australia. ${ }^{2}$ Royal Brisbane and Women's Hospital, Brisbane, Australia. ${ }^{3}$ Ghent University Hospital, Ghent, Belgium. ${ }^{4}$ Attikon University Hospital, Athens, Greece. ${ }^{5}$ Hospital Nord, Marseille, France. ${ }^{6}$ Centre Hospitalier Universitaire Bichat-Claude Bernard, AP-HP, Université Paris VII, Paris, France. ${ }^{7}$ Hospital Vall d'Hebron, Barcelona, Spain. ${ }^{8}$ St George's Healthcare NHS Trust and St George's University of London, London, England, UK

Received: 11 April 2012 Accepted: 28 June 2012

Published: 6 July 2012

\section{References}

1. Bagshaw SM, Uchino S, Bellomo R, Morimatsu H, Morgera S, Schetz M, Tan I, Bouman C, Macedo E, Gibney N, et al: Septic acute kidney injury in critically ill patients: clinical characteristics and outcomes. Clin J Am SoC Nephrol 2007, 2(3):431-439.

2. Hoste EA, Lameire NH, Vanholder RC, Benoit DD, Decruyenaere JM, Colardyn FA: Acute renal failure in patients with sepsis in a surgical ICU: predictive factors, incidence, comorbidity, and outcome. J Am Soc Nephrol 2003, 14(4):1022-1030.

3. Kollef MH, Sherman G, Ward S, Fraser VJ: Inadequate antimicrobial treatment of infections: a risk factor for hospital mortality among critically ill patients. Chest 1999, 115(2):462-474.

4. Kumar A, Roberts D, Wood KE, Light B, Parrillo JE, Sharma S, Suppes R, Feinstein D, Zanotti S, Taiberg $L$, et al: Duration of hypotension before initiation of effective antimicrobial therapy is the critical determinant of survival in human septic shock. Crit Care Med 2006, 34(6):1589-1596.

5. Marshall JC: Inflammation, coagulopathy, and the pathogenesis of multiple organ dysfunction syndrome. Crit Care Med 2001, 29(7 Suppl):S99-S106.

6. Uchino S, Kellum JA, Bellomo R, Doig GS, Morimatsu H, Morgera S, Schetz M, Tan I, Bouman C, Macedo E, et al: Acute renal failure in critically il patients: a multinational, multicenter study. JAMA 2005, 294(7):813-818.

7. Vincent JL, Rello J, Marshall J, Silva E, Anzueto A, Martin CD, Moreno R, Lipman J, Gomersall C, Sakr Y, et al: International study of the prevalence and outcomes of infection in intensive care units. JAMA 2009, 302(21):2323-2329.

8. Bernard GR, Vincent JL, Laterre PF, LaRosa SP, Dhainaut JF, Lopez-Rodriguez A, Steingrub JS, Garber GE, Helterbrand JD, Ely EW, et al: Efficacy and safety of recombinant human activated protein $\mathrm{C}$ for severe sepsis. $\mathrm{N}$ Engl J Med 2001, 344(10):699-709.

9. Lipman J, Udy AA, Roberts JA: Do we understand the impact of altered physiology, consequent interventions and resultant clinical scenarios in the intensive care unit? The antibiotic story. Anaesth Intensive Care 2011, 39:999-1000.

10. Pea F, Viale P, Furlanut M: Antimicrobial therapy in critically ill patients: review of pathophysiological conditions responsible for altered disposition and pharmacokinetic variability. Clin Pharmacokinet 2005 44(10):1009-1034.

11. Roberts JA, Lipman J: Antibacterial dosing in intensive care: pharmacokinetics, degree of disease and pharmacodynamics of sepsis. Clin Pharmacokinet 2006, 45(8):755-773.

12. Roberts JA, Kruger P, Paterson DL, Lipman J: Antibiotic resistance-what's dosing got to do with it? Crit Care Med 2008, 36(8):2433-2440.

13. Ariano RE, Nyhlen A, Donnelly JP, Sitar DS, Harding GK, Zelenitsky SA: Pharmacokinetics and pharmacodynamics of meropenem in febrile neutropenic patients with bacteremia. Ann Pharmacother 2005 . 39(1):32-38

14. Li C, Du X, Kuti JL, Nicolau DP: Clinical pharmacodynamics of meropenem in patients with lower respiratory tract infections. Antimicrob Agents Chemother 2007, 51(5):1725-1730.

15. McKinnon PS, Paladino JA, Schentag JJ: Evaluation of area under the inhibitory curve (AUIC) and time above the minimum inhibitory concentration ( $\mathrm{T}>\mathrm{MIC}$ ) as predictors of outcome for cefepime and ceftazidime in serious bacterial infections. Int J Antimicrob Agents 2008 31(4):345-351.

16. Sinnollareddy M, Peake SL, Roberts MS, Lipman J, Roberts JA: Using pharmacokinetics and pharmacodynamics to optimise dosing of antifungal agents in critically ill patients: a systematic review. Int Antimicrob Agents 2012, 39(1):1-10.

17. Sinnollareddy M, Peake SL, Roberts MS, Playford EG, Lipman J, Roberts JA: Pharmacokinetic evaluation of fluconazole in critically ill patients. Expert Opin Drug Metab Toxicol 2011, 7(11):1431-1440

18. Samtani MN, Flamm R, Kaniga K, Nandy P: Pharmacokineticpharmacodynamic-model-guided doripenem dosing in critically ill patients. Antimicrob Agents Chemother 2010, 54(6):2360-2364.

19. Roberts JA, Lipman J: Closing the loop - a colistin clinical study to confirm dosing recommendations from PK/PD modeling. Clin Infect Dis Off Publ Infect Dis Soc Am 2012, 54(12):1727-9.

20. Roberts JA, Norris R, Paterson DL, Martin JH: Therapeutic drug monitoring of antimicrobials. Br J Clin Pharmacol 2012, 73(1):27-36.

21. Udy AA, Roberts JA, Dewaele JJ, Paterson DL, Lipman J: What's behind the failure of emerging antibiotics in the critically ill? Understanding the impactof altered pharmacokinetics and augmented renal clearance. Int J Antimicrob Agents 2012, 39(6):455-7.

22. Roberts JA, Field J, Visser A, Whitbread R, Tallot M, Lipman J, Kirkpatrick CM: Using population pharmacokinetics to determine gentamicin dosing during extended daily diafiltration in critically ill patients with acute kidney injury. Antimicrob Agents Chemother 2010, 54(9):3635-3640.

23. Roberts JA, Kirkpatrick CM, Roberts MS, Robertson TA, Dalley AJ, Lipman J: Meropenem dosing in critically ill patients with sepsis and without renal dysfunction: intermittent bolus versus continuous administration? Monte Carlo dosing simulations and subcutaneous tissue distribution. J Antimicrob Chemother 2009, 64(1):142-150.

24. Roberts JA, Roberts MS, Robertson TA, Dalley AJ, Lipman J: Piperacillin penetration into tissue of critically ill patients with sepsis-bolus versus continuous administration? Crit Care Med 2009, 37(3):926-933.

25. Taccone FS, de Backer D, Laterre PF, Spapen H, Dugernier T, Delattre I, Wallemacq P, Vincent JL, Jacobs F: Pharmacokinetics of a loading dose of amikacin in septic patients undergoing continuous renal replacement therapy. Int J Antimicrob Agents 2011, 37(6):531-535.

26. Taccone FS, Laterre PF, Dugernier T, Spapen H, Delattre I, Wittebole X, De Backer D, Layeux B, Wallemacq P, Vincent JL, et al: Insufficient beta-lactam concentrations in the early phase of severe sepsis and septic shock. Crit Care 2010, 14(4):R126.

27. Bilgrami I, Roberts JA, Wallis SC, Thomas J, Davis J, Fowler S, Goldrick PB, Lipman J: Meropenem dosing in critically ill patients with sepsis recieving high volume continuous veno-venous hemofiltration. Antimicrob Agents Chemother 2010, 54(7):2974-2978.

28. Roberts DM, Roberts JA, Roberts MS, Liu X, Cole CL, Nair P, Lipman J, Bellomo R: Variability of antibiotic concentrations in critically ill patients receiving continuous renal replacement therapy - a multicentre pharmacokinetic study. Crit Care Med 2012, 40(5):1523-1528.

29. Roberts JA, Kirkpatrick CM, Roberts MS, Dalley AJ, Lipman J: First-dose and steady-state population pharmacokinetics and pharmacodynamics of piperacillin by continuous or intermittent dosing in critically ill patients with sepsis. Int J Antimicrob Agents 2010, 35(2):156-163.

30. Lipman J, Wallis SC, Rickard C: Low plasma cefepime levels in critically ill septic patients: pharmacokinetic modeling indicates improved troughs with revised dosing. Antimicrob Agents Chemother 1999, 43(10):2559-2561

31. Lipman J, Wallis SC, Rickard CM, Fraenkel D: Low cefpirome levels during twice daily dosing in critically ill septic patients: pharmacokinetic modelling calls for more frequent dosing. Intensive Care Med 2001, 27(2):363-370.

32. Ulldemolins M, Roberts JA, Wallis SC, Rello J, Lipman J: Flucloxacillin dosing in critically ill patients with hypoalbuminaemia: special emphasis on unbound pharmacokinetics. J Antimicrob Chemother 2010, 65(8):1771-1778.

33. Roberts JA, Ulldemolins M, Roberts MS, McWhinney B, Ungerer J, Paterson $\mathrm{DL}$, Lipman J: Therapeutic drug monitoring of beta-lactams in critically ill patients: proof of concept. Int J Antimicrob Agents 2010, 36(4):332-339.

34. Vincent IL, de Mendonca A Cantraine F, Moreno R, Takala J, Suter PM, Sprung $\mathrm{CL}$, Colardyn F, Blecher S: Use of the SOFA score to assess the incidence of organ dysfunction/failure in intensive care units: results of a multicenter, prospective study. Working group on "sepsis-related problems" of the European Society of Intensive Care Medicine. Crit Care Med 1998, 26(11):1793-1800.

35. Knaus WA, Draper EA, Wagner DP, Zimmerman JE: APACHE II: A severity of disease classification system. Crit Care Med 1985, 13:818-829. 
36. Lisboa T, Diaz E, Sa-Borges M, Socias A, Sole-Violan J, Rodriguez A, Rello J: The ventilator-associated pneumonia PIRO score: a tool for predicting ICU mortality and health-care resources use in ventilator-associated pneumonia. Chest 2008, 134(6):1208-1216.

37. Turnidge JD: The pharmacodynamics of beta-lactams. Clin Infect Dis 1998, 27(1):10-22.

38. Patel K, Roberts JA, Lipman J, Tett SE, Deldot ME, Kirkpatrick CM: Population pharmacokinetics of fluconazole in critically ill patients receiving continuous venovenous hemodiafiltration: using Monte Carlo simulations to predict doses for specified pharmacodynamic targets. Antimicrob Agents Chemother 2011, 55(12):5868-5873.

39. Faul F, Erdfelder E, Lang AG, Buchner A: G*Power 3: a flexible statistical power analysis program for the social, behavioral, and biomedical sciences. Behav Res Methods 2007, 39(2):175-191.

40. Roberts JA, Kirkpatrick CM, Lipman J: Monte Carlo simulations: maximizing antibiotic pharmacokinetic data to optimize clinical practice for critically ill patients. J Antimicrob Chemother 2011, 66(2):227-231.

doi:10.1186/1471-2334-12-152

Cite this article as: Roberts et al.: DALI: Defining Antibiotic Levels in Intensive care unit patients: a multi-centre point of prevalence study to determine whether contemporary antibiotic dosing for critically ill patients is therapeutic. BMC Infectious Diseases 2012 12:152.

\section{Submit your next manuscript to BioMed Central and take full advantage of:}

- Convenient online submission

- Thorough peer review

- No space constraints or color figure charges

- Immediate publication on acceptance

- Inclusion in PubMed, CAS, Scopus and Google Scholar

- Research which is freely available for redistribution 